\title{
Formation of secondary electron cascades in single-crystalline plasma-deposited diamond upon exposure to femtosecond $\mathrm{X}$-ray pulses
}

\author{
M. Gabrysch, ${ }^{1}$ E. Marklund, ${ }^{2}$ J. Hajdu, ${ }^{2,3}$ D. J. Twitchen, ${ }^{4}$ J. Rudati, ${ }^{3}$ A. M. Lindenberg, ${ }^{5,6}$ \\ C. Caleman, ${ }^{2}$ R. W. Falcone ${ }^{7}$ T. Tschentscher ${ }^{8}$ K. Moffat, ${ }^{9}$ P. H. Bucksbaum, ${ }^{6}$ \\ J. Als-Nielsen, ${ }^{10}$ A. J. Nelson, ${ }^{11}$ D. P. Siddons, ${ }^{12}$ P. J. Emma, ${ }^{3}$ P. Krejcik, ${ }^{3}$ H. Schlarb, ${ }^{3}$ \\ J. Arthur, ${ }^{3}$ S. Brennan, ${ }^{3}$ J. Hastings, ${ }^{3}$ and J. Isberg ${ }^{1, a)}$ \\ ${ }^{1}$ Division of Electricity, Uppsala University, Box 534, 75121 Uppsala, Sweden \\ ${ }^{2}$ ICM Molecular Biophysics, Biomedical Centre, Uppsala University, Box 596, 75124 Uppsala, Sweden \\ ${ }^{3}$ Stanford Linear Accelerator Center, Menlo Park, California 94025, USA \\ ${ }^{4}$ Element Six Ltd, King's Ride Park, Ascot, Berkshire, SL5 8BP, United Kingdom \\ ${ }^{5}$ Department of Materials Science and Engineering, Stanford University, Stanford, \\ California 94305, USA \\ ${ }^{6}$ PULSE Center, Stanford Linear Accelerator Center, Menlo Park, California 94025, USA \\ ${ }^{7}$ Department of Physics, University of California, Berkeley, California 94720, USA \\ ${ }^{8}$ Deutsches Elektronen-Synchrotron DESY, Notkestrasse 85, 22607 Hamburg, Germany \\ ${ }^{9}$ Consortium for Advanced Radiation Sources, The University of Chicago, Chicago, Illinois 60637, USA \\ ${ }^{10}$ Niels Bohr Institute, Copenhagen University, 2100 Copenhagen $\emptyset$, Denmark \\ ${ }^{11}$ Lawrence Livermore National Laboratory, Livermore, California 94550, USA \\ ${ }^{12}$ National Synchrotron Light Source, Brookhaven National Laboratory, Upton, New York 11973, USA
}

(Received 11 December 2007; accepted 1 January 2008; published online 24 March 2008)

\begin{abstract}
Secondary electron cascades were measured in high purity single-crystalline chemical vapor deposition (CVD) diamond, following exposure to ultrashort hard x-ray pulses (140 fs full width at half maximum, $8.9 \mathrm{keV}$ energy) from the Sub-Picosecond Pulse Source at the Stanford Linear Accelerator Center. We report measurements of the pair creation energy and of drift mobility of carriers in two CVD diamond crystals. This was done for the first time using femtosecond x-ray excitation. Values for the average pair creation energy were found to be $12.17 \pm 0.57$ and $11.81 \pm 0.59 \mathrm{eV}$ for the two crystals, respectively. These values are in good agreement with recent theoretical predictions. The average drift mobility of carriers, obtained by the best fit to device simulations, was $\mu_{h}=2750 \mathrm{~cm}^{2} / \mathrm{V}$ s for holes and was $\mu_{e}=2760 \mathrm{~cm}^{2} / \mathrm{V} \mathrm{s}$ for electrons. These mobility values represent lower bounds for charge mobilities due to possible polarization of the samples. The results demonstrate outstanding electric properties and the enormous potential of diamond in ultrafast x-ray detectors. (C) 2008 American Institute of Physics.
\end{abstract}

[DOI: $10.1063 / 1.2890158$ ]

\section{INTRODUCTION}

Due to the extraordinary material properties of diamond, it is attracting much attention in ionizing radiation detector applications. Diamond exhibits a wide band gap $(5.47 \mathrm{eV})$ allowing for detectors with low leakage currents and low noise levels for operation over a wide temperature range. In addition, the low atomic number of carbon $(Z=6)$ makes diamond near tissue equivalent, which is important in biomedical applications. The strong $s p^{3}$ carbon-carbon bond that makes diamond the hardest of all materials also results in a very high damage threshold for ionizing radiation. ${ }^{1}$ This makes diamond films suitable for particle detector applications without a significant degradation in performance from radiation damage. In addition, single-crystalline chemical vapor deposition (SC-CVD) diamond exhibits a high saturation drift velocity, high carrier mobilities, ${ }^{2,3}$ and high charge collection efficiency with good spatial homogeneity. ${ }^{4}$

The maximum resolution, e.g., in imaging biological materials, that can be achieved in x-ray diffraction is today

\footnotetext{
${ }^{a)}$ Author to whom correspondence should be addressed. Electronic mail: jan.isberg@angstrom.uu.se.
}

limited by damage. Ultrashort high-intensity x-ray pulses from sources like x-ray free electron lasers, which currently are under development, are expected to extend this limit significantly. ${ }^{5}$ Diamond could be used for continuous beam monitoring in such sources due to its high resilience to radiation.

X-ray photons interact with a material mainly via the photoelectric effect. In light elements, such as carbon, the emission of an energetic photoelectron from the $K$ shell is followed by the emission of a less energetic Auger electron. These electrons then propagate through the material and thermalize, initiating a cascade of secondary electrons in macroscopic samples. The cascades play a major role in the ionization dynamics of samples, and contribute significantly to radiation damage. In a semiconductor, such as diamond, the cascades result in the excitation of electrons from the valence band to the conduction band-or expressed differently-in the formation of electron-hole pairs. These pairs will ultimately recombine in the sample, or alternatively, they can be collected by an applied electric field at electrodes, with near $100 \%$ collection efficiency in high purity semiconductor materials. 
Until recently, the situation for the determination of the average pair creation energy $\varepsilon_{\mathrm{pc}}$ in diamond was comparable to that of silicon before the 1970s. Due to poor sample quality there was a vast amount of different values in the literature. For natural diamond, values from $13 \mathrm{eV}$ (Refs. 6 and 7) up to $18.5 \mathrm{eV}$ (Ref. 8) and even $24.5 \mathrm{eV}$ were reported. ${ }^{9}$ Poor sample quality results in short carrier lifetime, and prevents complete extraction of all charge created in the sample. As a consequence, the pair creation energy appears to be higher. Improvements in the growth of high purity SC-CVD diamonds have opened up new possibilities for accurate measurements. However, even for CVD diamonds the reported values in the literature vary between roughly 12 and $13.5 \mathrm{eV}$ (e.g., $12.8 \mathrm{eV}$ from $\alpha$-particle measurements. ${ }^{10}$ ) In addition there exists a remarkable correlation between the pair creation energy $\left(\varepsilon_{\mathrm{pc}}\right)$ and the band gap energy $\left(E_{g}\right)$ that different phenomenological models have tried to explain for the past four decades. ${ }^{11-13}$ Many semiconductors (e.g., Ge, $\mathrm{Si}$, and $\mathrm{GaAs})$ obey the relation $\varepsilon_{\mathrm{pc}}=\frac{14}{5} E_{g}+0.6 \mathrm{eV},{ }^{13}$ which for diamond would imply $\varepsilon_{\mathrm{pc}}=15.9 \mathrm{eV}$. There is no compelling reason why different excitation sources ( $\alpha$ /heavy ions, $\beta, \gamma / \mathrm{x}$-ray) should lead to exactly the same $\varepsilon_{\mathrm{pc}}$ since e.g., $\alpha$-particles, besides interacting with electrons, can also transfer energy to the lattice, e.g., by the creation of defects. Therefore, it is of great importance to measure this material constant with all kinds of excitation sources and to compare these values.

Model calculations of the evolution of secondary electron cascades in diamond have yielded predictions that can be tested experimentally. At the same time they lead to an improved understanding of secondary emission rates and radiation damage. ${ }^{14}$ This can be applied to other covalent carbon structures, including biomolecules. Ziaja et al. ${ }^{15-17}$ have used Monte Carlo simulations to calculate the temporal evolution of electron cascades showing that the emission of secondary electrons approaches saturation within about $100 \mathrm{fs}$. Simulations using a unified model, ${ }^{17}$ based on an empirical model $^{18}$ for higher electron energies $(\gtrsim 100 \mathrm{eV})$ and first principles calculations ${ }^{19}$ at lower energies $(\lessgtr 10 \mathrm{eV})$, give precise values for the pair creation energy that can be compared with experiments.

In this paper we describe measurements of the pair creation energy in diamond using two detectors made from high purity SC-CVD diamond illuminated by ultrashort $8.9 \mathrm{keV}$ x-ray pulses from the Sub-Picosecond Pulse Source (SPPS) at the Stanford Linear Accelerator Center (SLAC). This source delivers pulses for excitation in the same range of parameters as used for the simulations in Ziaja et al., ${ }^{17}$ enabling direct comparison without additional assumptions, such as independency of $\varepsilon_{\mathrm{pc}}$ on type or energy of the exciting radiation, etc.

The paper is structured as follows. Section II presents briefly the properties of SPPS and is followed by a description of our diamond detectors. In Sec. III we present and discuss the results on pair creation energy. The ultrashort x-ray pulses have also been used to create electron-hole pairs for drift mobility measurements in our detectors. This is described in Sec. IV. Finally, Sec. V contains the conclusions.
TABLE I. Properties and measured average energy per created electron-hole pair of the diamond detectors including the reference PIPS diode. The absorption coefficients are taken from Ref. 27 and correspond to the denoted material, its thickness, and the photon energy of $E_{\gamma}=8.90 \pm 0.05 \mathrm{keV}$.

\begin{tabular}{lccc}
\hline \hline & PIPS diode & Sample 1 & Sample 2 \\
\hline Material & Silicon $(\mathrm{Si})$ & SC-CVD diamond (D) \\
Thickness $(\mu \mathrm{m})$ & $304 \pm 2$ & $528 \pm 5$ & $690 \pm 5$ \\
Absorption $A$ for $E=8.9 \mathrm{keV}$ & $0.960 \pm 0.003$ & $0.443 \pm 0.009$ & $0.534 \pm 0.009$ \\
Slope $\lambda$ & $12 \pm 0.2$ & $1.651 \pm 0.004$ & $2.051 \pm 0.006$ \\
Pair creation energy $\varepsilon_{\mathrm{pc}}(\mathrm{eV})$ & $3.63 \pm 0.03$ & $12.17 \pm 0.57$ & $11.81 \pm 0.59$ \\
\hline \hline
\end{tabular}

\section{DESCRIPTION OF THE RADIATION SOURCE AND THE DETECTORS}

The SPPS at SLAC is a linac-based light source capable of producing ultrashort hard x-ray pulses. Electrons are accelerated over $3 \mathrm{~km}$ up to an energy of $28.5 \mathrm{GeV}$, and injected into a $2.5 \mathrm{~m}$ long undulator where radiation is emitted. $^{20,21}$ Efficient bunch compression allows pulse durations on a subpicosecond time scale. Both theoretical calculations and hands-on measurements determine the average bunch length to be $80 \mathrm{fs}$ full width at half maximum under optimal conditions, producing $\mathrm{x}$-ray pulses of similar durations. ${ }^{21-23}$ SPPS produces up to $2 \times 10^{7}$ photons/pulse at a $10 \mathrm{~Hz}$ repetition rate with an energy of several $\mathrm{keV}^{20,21}$ The photon energy in the experiments described below was determined by measuring the Bragg angle of a bismuth crystal. This yielded a photon energy of $8.90 \pm 0.05 \mathrm{keV}$. The width of the unfocused beam is of the order of $1 \mathrm{~mm}$, but can be focused to less than half a millimeter across with a beryllium $(\mathrm{Be})$ lens which reduces the number of transmitted photons by roughly an order of magnitude. ${ }^{24}$ In the past, SPPS has successfully been utilized to assess ultrafast processes in crystalline materials. ${ }^{21-23}$ The SPPS undulator has, at the time of writing, been removed from SLAC to make room for the upcoming Linac Coherent Light Source.

The diamond detectors used in the experiments were manufactured from SC-CVD diamond plates produced and supplied by E6. The material was synthesized using a microwave plasma-assisted CVD reactor operating at a frequency of $2.45 \mathrm{GHz}$. Homoepitaxial CVD diamond was deposited on especially prepared high-pressure high-temperature (HPHT) synthetic diamond substrates. A pregrowth etch phase was followed by epitaxial overgrowth under conditions of high purity. After synthesis the high purity epitaxial overlayer was first separated from its HPHT diamond substrate by a laser cutting technique, and then polished to give a freestanding high purity plate. From electron paramagnetic resonance measurements, the concentration of nitrogen impurities in this material is known to be below 5 $\times 10^{14} \mathrm{~cm}^{-3}$. Both sides of the sample were metallized by sputtering, using first $\mathrm{Ti}$ and then $\mathrm{Al}$ in a physical vapor deposition system. The contacts were patterned by means of standard optical lithography techniques to form a semitransparent mesh pattern, $4 \mathrm{~mm}$ in diameter, on the (100) surfaces. Al was etched with $\mathrm{H}_{3} \mathrm{PO}_{4}: \mathrm{CH}_{3} \mathrm{COOH}: \mathrm{NO}_{3}$, and $\mathrm{Ti}$ was etched with a weak solution of $\mathrm{HF}: \mathrm{HNO}_{3}$ in water. The plates were annealed for 


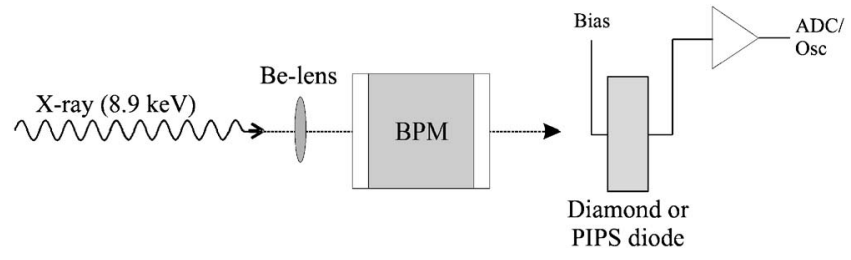

FIG. 1. Experimental setup. X-rays are first focused by a beryllium lens, then pass the BPM and penetrate a diamond sample or the PIPS diode for reference measurements.

$15 \mathrm{~min}$ at $600{ }^{\circ} \mathrm{C}$ in argon and mounted in holders. Table I lists key parameters for these crystals and for a passivated implanted planar silicon (PIPS) diode detector used as reference.

\section{MEASUREMENT OF INDUCED CHARGE BY FEMTOSECOND X-RAY PULSES}

\section{A. Experimental setup}

Figure 1 shows the scheme of the experiment. Our aim was to measure the extracted charge per x-ray pulse in order to determine the average energy for creating an electron-hole pair in diamond. Measurements are performed on our two SC-CVD diamonds described above (referred to as "Samples 1 and 2" in Table I). A diamond sample is mounted with the (100) faces perpendicular to the incident beam and illuminated with ultrashort x-ray pulses $[\lesssim 140 \mathrm{fs}, 8.9 \mathrm{keV}$, $(1.5 \pm 0.2) \times 10^{6}$ photons/shot $]$ at a repetition rate of $10 \mathrm{~Hz}$. Nonoptimal bunch compression and limited temporal resolution due to the response time of the electro-optic crystals used for beam profiling cause the pulse length to exceed the $80 \mathrm{fs}$ previously reported. ${ }^{21-23}$ The generated charge is collected at the electrodes by applying a dc bias (values ranging from 0 to $300 \mathrm{~V}$ ). The charge collection efficiency in this material is known from previous measurements to be close to $100 \%$ with a charge collection distance (at $1 \mathrm{~V} / \mu \mathrm{m}$ ) on the order of millimeters and even centimeters. ${ }^{2,25}$ The extracted charge is measured by a charge sensitive preamplifier. The amplifier, based on a Cremat CR-110 circuit, is especially designed for this purpose. The signal is then shaped with a Gaussian shaping amplifier Cremat CR-160 and sampled by an Ortec AD413A Quad 8k analog-to-digital converter

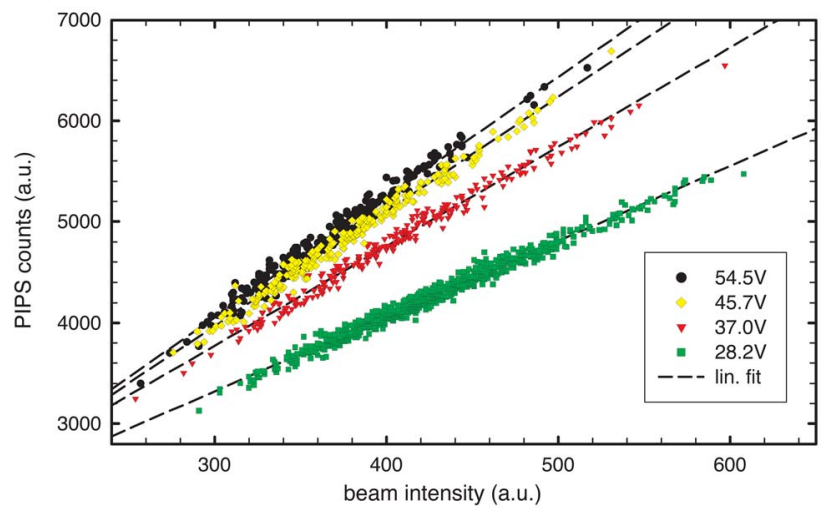

FIG. 2. (Color online) Plot showing the linear relation between the response of PIPS diode and BPM for different bias voltages over the diode. Data for 300-1000 pulses per bias voltage.

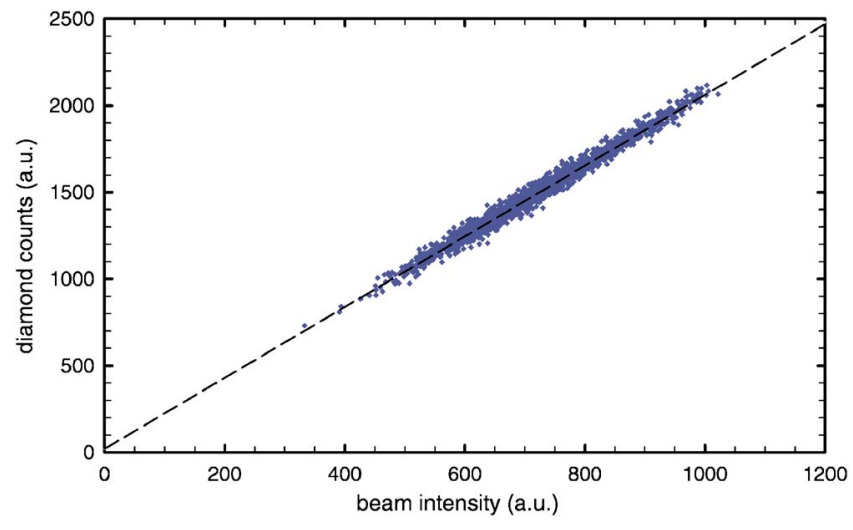

FIG. 3. (Color online) Plot showing the linear relation between the response of diamond detector 2 and the BPM. Data for 3300 pulses with saturated detector (bias $>200 \mathrm{~V}$ ).

(ADC). The sample and preamplifier are carefully shielded to avoid electromagnetic (EM) interference from surrounding equipment. An argon-filled ionization chamber-beam positioning monitor (BPM) - is located upstream from the sample. The BPM can be used to monitor the intensity of individual pulses with a response proportional to the number of photons. Its signal is also read out by the Ortec ADC.

For reference purpose, the diamond sample can be replaced by a PIPS diode, Canberra FD 300-20-300 RM. The PIPS diode is connected to exactly the same chain of amplifiers as the diamond sample and sufficient bias voltage is applied in order to assure saturation of the diode. The collection efficiency of the PIPS diode is very close to $100 \%$.

\section{B. Results}

Figure 2 plots PIPS counts versus beam intensity, and shows that these are linearly related for different bias voltages on the PIPS. The figure also shows that the PIPS diode is nearly saturated at a bias of about $50 \mathrm{~V}$ and the corresponding linear fit is the one entering the calculations in what follows (see Table I).

The response of the diamond detector is also linear versus the BPM, as exemplified in Fig. 3. Since it is not possible to measure simultaneously on the diamond samples and the PIPS diode (needed for reference) we use linear fits in order to compare measurements.

The measured count rate $N$ for both the diamond sample and the silicon PIPS diode is directly proportional to the collected charge $Q_{\text {coll }}$ with a constant of proportionality $\alpha$

$$
N=\alpha Q_{\text {coll }} .
$$

As can be seen in Fig. 4 the collected charge becomes independent of the bias voltage, above a certain threshold, indicating a near $100 \%$ collection efficiency. ${ }^{\mathrm{a}}$

The created charge $Q$ is proportional to the photon energy $E_{\gamma}$ and the number of absorbed photons $I$, but inversely proportional to the average creation energy for an electronhole pair $\varepsilon_{\mathrm{pc}}$. The number of absorbed photons $I$ is simply

${ }^{\mathrm{a}}$ Lower efficiency would lead to a higher average pair creation energy. That is why our result can at least be seen as an upper limit. 


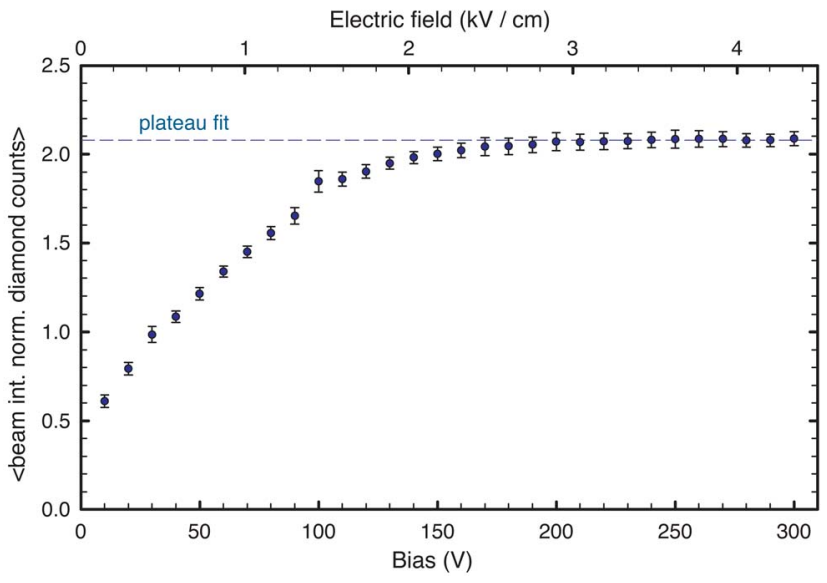

FIG. 4. (Color online) Mean value (over 300 pulses/bias) of BPMnormalized ADC counts for Sample 2 as a function of electric field and bias, respectively.

the total number of photons $I_{0}$ times the absorption $A$. Since the BPM-count rate $N_{\mathrm{BPM}}$ is also proportional to the total photon flux $I_{0}$ (by a factor $\beta$ ) we write

$$
Q_{\mathrm{coll}}=Q=I \frac{E_{\gamma}}{\varepsilon_{\mathrm{pc}}}=A I_{0} \frac{E_{\gamma}}{\varepsilon_{\mathrm{pc}}}=A \frac{N_{\mathrm{BPM}}}{\beta} \frac{E_{\gamma}}{\varepsilon_{\mathrm{pc}}} .
$$

Thus, the overall dependence between $N$ and $N_{\text {BPM }}$ reads

$$
N=\alpha A \frac{N_{\mathrm{BPM}}}{\beta} \frac{E_{\gamma}}{\varepsilon_{\mathrm{pc}}} \equiv \lambda N_{\mathrm{BPM}},
$$

with

$$
\lambda \equiv \frac{\alpha A E_{\gamma}}{\beta \varepsilon_{\mathrm{pc}}}
$$

being the proportionality factor.

This factor is given by the slope of the linear fit in a (Si/diamond, BPM) plot. Thus, by taking the quotient of diamond (D) and silicon $(\mathrm{Si})$ related slopes, material independent quantities cancel out and we are only left with the relative absorption $I / I_{0}$ and the average energy per created electron-hole pair $^{\mathrm{b}}$. Therefore, we can write

$$
\varepsilon_{\mathrm{pc}}^{\mathrm{D}}=\frac{\lambda_{\mathrm{Si}}}{\lambda_{\mathrm{D}}} \frac{A_{\mathrm{D}}}{A_{\mathrm{Si}}} \varepsilon_{\mathrm{pc}}^{\mathrm{Si}}
$$

Knowing the pair creation value for silicon $(3.63 \mathrm{eV} \pm 0.03 \mathrm{eV})$ at room temperature, ${ }^{26}$ we obtain the corresponding values for the diamond sample. The results are presented in Table I.

\section{MEASUREMENT OF DRIFT MOBILITY}

\section{A. Experimental setup}

For these measurements sample 1 was rotated by $90 \mathrm{deg}$ and mounted with the (100) faces parallel to the beam and illuminated through a narrow slit $(150 \mu \mathrm{m}$ wide), onto one edge with ultrashort x-ray pulses $(\lesssim 140 \mathrm{fs}, 8.9 \mathrm{keV})$ at a repetition rate of $10 \mathrm{~Hz}$. More than $95 \%$ of the photons get

${ }^{b}$ Note that actually an implicit dependency on the photon energy remains through the magnitudes of the absorption coeffieicts.

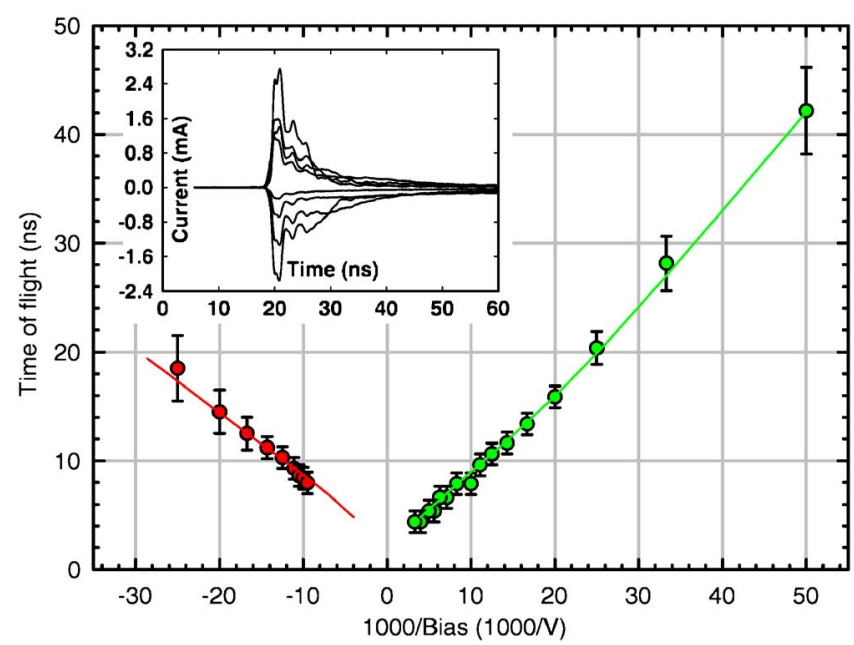

FIG. 5. (Color online) Transit times for holes (neg. bias) and electrons (pos. bias) vs inverse bias voltage. Solid lines show best fits to device simulations (see text). The inset shows current traces for different bias voltages as recorded by the oscilloscope.

absorbed and generate electron-hole pairs in close proximity to one of the contacts. By applying a dc bias across the sample, holes or electrons (depending on bias polarity) traverse the sample and are collected at the opposite contact. The dc bias is varied in steps between -300 and $+300 \mathrm{~V}$. The resulting current is measured by a broadband current amplifier located near the sample. The amplifier, based on a MAX4223 operation amplifier, is especially designed for this purpose. The sample and amplifier are carefully shielded to avoid EM interference. The signal is recorded using a DSO81304A Infiniium oscilloscope from Agilent Technologies with $13 \mathrm{GHz}$ bandwidth. For each bias voltage, the signal is averaged over 64 pulses to reduce the influence of fluctuations in beam intensity between consecutive pulses.

\section{B. Results}

The temporal profile of the current is expected to be dependent on the amount of space charge generated by the $\mathrm{x}$-ray pulse. This follows because a high concentration of space charge will appreciably affect the electric field distribution across the sample. In addition, trapping centers may cause a more or less permanent polarization of the sample. The space charge effect becomes substantial if the injected charge $Q$ is approximately equal to or larger than $C U$, where $C$ is the sample capacitance and $U$ the bias voltage. In this experiment the charge created is about $10 \mathrm{pC} / \mathrm{pulse}$, the sample capacitance is $0.35 \mathrm{pF}$, and bias voltages in the range $10-300 \mathrm{~V}$ have been used. Thus, the experiment is conducted in the "difficult" region $Q \approx C U$, requiring comparison with device simulations in order to interpret data. Examples of electric current traces for both positive and negative bias polarities are shown in the inset in Fig. 5. The signal exhibits some ringing, apparently due to some impedance mismatch in the circuit. Nevertheless the signal can be seen to display a characteristic sharp drop at a certain (biasdependent) time characteristic of a carrier transient across the sample. Measured transit times versus inverse bias are shown in Fig. 5. 


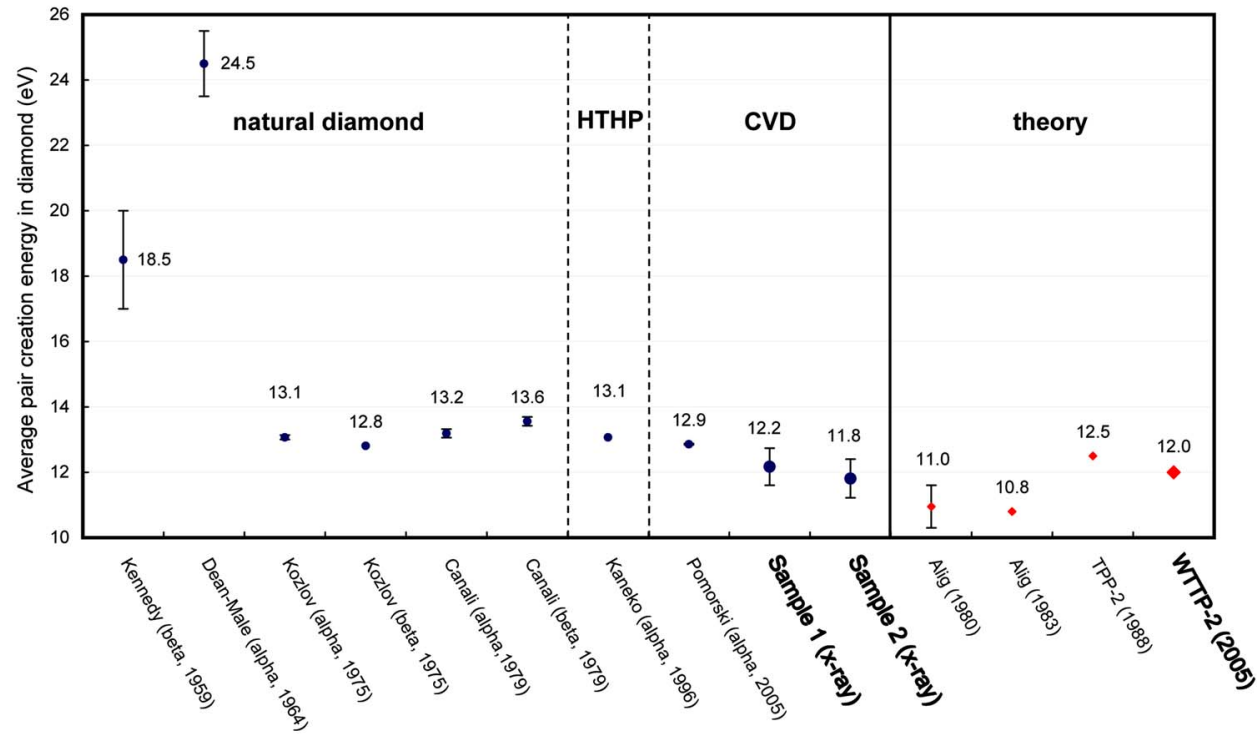

FIG. 6. (Color online) Average pair creation energy in diamond obtained from experiments (left side, blue) and theory (right side, red). References: natural diamond (Refs. 6-9) HTHP (Ref. 27), CVD (Ref. 10), and theoretical treatment (Ref. 17) and therein.
By assuming a linear relation between drift velocity $v$ and electric field $E$ (i.e., a constant mobility $\mu$ with $v=\mu E$; this is reasonable for the low bias voltages in this experiment) and by neglecting polarization effects due to trapping, the data can be fitted well to device simulations based on the drift-diffusion and Poisson equations. Least-squares fits of device simulations to experimental data are indicated by the solid lines in Fig. 5, with $\mu_{h}=2750$ and $\mu_{e}=2760 \mathrm{~cm}^{2} / \mathrm{V} \mathrm{s}$. However, although it is possible in principle to measure the amount of trapped charge in this type of experiment, ${ }^{27}$ this was not possible with the relatively low signal to noise ratio achieved. Thus it cannot be excluded that polarization effects are important. Homogeneous polarization of the sample will normally increase the transit time and therefore the drift mobility values quoted above must only be regarded as lower bounds.

\section{DISCUSSION AND CONCLUSIONS}

Measured values for the average pair creation energy in diamond $12.17 \pm 0.57$ and $11.81 \pm 0.59 \mathrm{eV}$ can be directly compared to theoretical predictions from the Monte Carlo simulations of Ziaja et al. ${ }^{17}$ In their paper two different models are used. The TPP-2 model, which yields a pair creation energy of $12.5 \mathrm{eV}$, and the more complex WTPP-2 model, which yields a pair creation energy of $12.0 \mathrm{eV}$. As can be seen in Fig. 6 our data are in good agreement with predictions based on the WTPP-2 model. This shows that an accurate treatment of low energy cross sections is needed to agree with experimental results.

Even though our results have tighter error bounds than most previous results, there is still a big error coming to a large extent from the reference measurement with the PIPS diode. By simply improving statistics one could decrease the relative error from $4 \%$ down to $2.5 \%$. In order to decrease the error margin even more would involve direct absorption measurements for the samples which could also be done, e.g., by using two identical ionization chambers with the diamond detector in between.
By measuring the transit time of carriers through a sample at different bias and comparing with device simulations it was also possible to measure low-field drift mobilities of holes and electrons, using the SPPS as excitation source. The best fit to data was obtained for $\mu_{h}=2750$ and $\mu_{e}=2760 \mathrm{~cm}^{2} / \mathrm{V} \mathrm{s}$. However, with the relatively low signal to noise ratio achieved in this experiment it cannot be excluded that strong polarization results in an inhomogeneous electric field distribution in the detector. Therefore these mobility values are lower bounds only.

\section{ACKNOWLEDGMENTS}

Part of this research was supported by the U.S. Department of Energy, Office of Basic Energy Science through direct support for the SPPS, as well as individual investigators and SSRL, a national user facility operated by Stanford University. Additional support for the construction of SPPS was provided by Uppsala University and the Swedish Research Council. Further support by the Ångpanneföreningens Foundation for Research and Development is kindly acknowledged. We are also thankful to Sebastien Boutet for his assistance at SPPS. M.G. and E.M. contributed equally to the paper.

${ }^{1}$ P. Bergonzo, A. Brambilla, D. Tromson, C. Mer, B. Guizard, F. Foulon, and V. Amosov, Diamond Relat. Mater. 10, 631 (2001).

${ }^{2}$ J. Isberg, J. Hammersberg, E. Johansson, T. Wikström, D. J. Twitchen, A. J. Whitehead, S. E. Coe, and G. A. Scarsbrook, Science 297, 1670 (2002).

${ }^{3}$ J. Isberg, A. Lindblom, A. Tajani, and D. J. Twitchen, Phys. Status Solidi A 1-5, 2194 (2005).

${ }^{4}$ A. Lohstroh, P. J. Sellin, S. G. Wang, A. W. Davies, J. Parkin, R. W. Martin, and P. R. Edwards, Appl. Phys. Lett. 90, 102111 (2007).

${ }^{5}$ R. Neutze, R. Wouts, D. van der Spoel, E. Weckert, and J. Hajdu, Nature (London) 406, 752 (2000).

${ }^{6}$ C. Canali, E. Gatti, S. F. Kozlov, P. F. Manfredi, C. Manfredotti, F. Nava, and A. Quirini, Nucl. Instrum. Methods 160, 73 (1979).

${ }^{7}$ S. F. Kozlov, R. Stuck, M. Hage-Ali, and P. Siffert, IEEE Trans. Nucl. Sci. 22, 160 (1975).

${ }^{8}$ P. J. Kennedy, Proc. R. Soc. London, Ser. A 253, 37 (1959).

${ }^{9}$ P. J. Dean and J. C. Male, J. Phys. Chem. Solids 22, 311 (1964).

${ }^{10}$ M. Pomorski, E. Berdermann, M. Ciobanu, A. Martemyianov, P. Moritz, M. Rebisz, and B. Marczewska, Phys. Status Solidi A 202, 2199 (2005).

${ }^{11}$ C. A. Klein, J. Appl. Phys. 39, 2029 (1968). 
${ }^{12}$ R. C. Alig and S. Bloom, Phys. Rev. Lett. 35, 1522 (1975).

${ }^{13}$ A. Owens and A. Peacock, Nucl. Instrum. Methods Phys. Res. A 531, 18 (2004).

${ }^{14}$ M. Bergh, N. Tîmneanu, and D. van der Spoel, Phys. Rev. E 70, 051904 (2004).

${ }^{15}$ B. Ziaja, D. van der Spoel, A. Szöke, and J. Hajdu, Phys. Rev. B 64, 214104 (2001)

${ }^{16}$ B. Ziaja, A. Szöke, D. van der Spoel, and J. Hajdu, Phys. Rev. B 66, 024116 (2002).

${ }^{17}$ B. Ziaja, R. A. London, and J. Hajdu, J. Appl. Phys. 97, 064905 (2005).

${ }^{18}$ S. Tanuma, C. J. Powell, and D. R. Penn, Surf. Interface Anal. 11, 577 (1988).

${ }^{19}$ T. Watanabe, M. Irie, T. Teraji, T. Ito, Y. Kamakura, and K. Taniguchi, Jpn. J. Appl. Phys., Part 2 40, L715 (2001).

${ }^{20}$ P. Krejcik, F.-J. Decker, P. Emma, K. Hacker, L. Hendrickson, C. L. O'Connell, H. Schlarb, H. Smith, and M. Stanek, in 2003 Particle Accelerator Conference, Portland, OR, 2003, edited by J. Chew, P. Lucas, and S. Webber (IEEE, Piscataway, NJ, 2003), p. 423.

${ }^{21}$ A. M. Lindenberg, J. Larsson, K. Sokolowski-Tinten, K. J. Gaffney, C. Blome, O. Synnergren, J. Sheppard, C. Caleman, A. G. MacPhee, D. Weinstein, D. P. Lowney, T. K. Allison, T. Matthews, R. W. Falcone, A. L. Cavalieri, D. M. Fritz, S. H. Lee, P. H. Bucksbaum, D. A. Reis, J. Rudati, P. H. Fuoss, C. C. Kao, D. P. Siddons, R. Pahl, J. Als-Nielsen, S. Duesterer, R. Ischebeck, H. Schlarb, H. Schulte-Schrepping, Th. Tschentscher, J. Schneider, D. von der Linde, O. Hignette, F. Sette, H. N. Chapman, R. W. Lee, T. N. Hansen, S. Techert, J. S. Wark, M. Bergh, G. Huldt, D. van der Spoel, N. Timneanu, J. Hajdu, R. A. Akre, E. Bong, P. Krejcik, J. Arthur, S. Brennan, K. Luening, and J. B. Hastings, Science 308, 392 (2005).
${ }^{22}$ K. J. Gaffney, A. M. Lindenberg, J. Larsson, K. Sokolowski-Tinten, C. Blome, O. Synnergren, J. Sheppard, C. Caleman, A. G. MacPhee, D. Weinstein, D. P. Lowney, T. Allison, T. Matthews, R. W. Falcone, A. L. Cavalieri, D. M. Fritz, S. H. Lee, P. H. Bucksbaum, D. A. Reis, J. Rudati, A. T. Macrander, P. H. Fuoss, C. C. Kao, D. P. Siddons, R. Pahl, K. Moffat, J. Als-Nielsen, S. Duesterer, R. Ischebeck, H. Schlarb, H. SchulteSchrepping, J. Schneider, D. von der Linde, O. Hignette, F. Sette, H. N. Chapman, R. W. Lee, T. N. Hansen, J. S. Wark, M. Bergh, G. Huldt, D. van der Spoel, N. Timneanu, J. Hajdu, R. A. Akre, E. Bong, P. Krejcik, J. Arthur, S. Brennan, K. Luening, and J. B. Hastings, Phys. Rev. Lett. 95, 125701 (2005).

${ }^{23}$ D. M. Fritz, D. A. Reis, B. Adams, R. A. Akre, J. Arthur, C. Blome, P. H. Bucksbaum, A. L. Cavalieri, S. Engemann, S. Fahy, R. W. Falcone, P. H. Fuoss, K. J. Gaffney, M. J. George, J. Hajdu, M. P. Hertlein, P. B. Hillyard, M. Horn-von Hoegen, M. Kammler, J. Kaspar, R. Kienberger, P. Krejcik, S. H. Lee, A. M. Lindenberg, B. McFarland, D. Meyer, T. Montagne, É. D. Murray, A. J. Nelson, M. Nicoul, R. Pahl, J. Rudati, H. Schlarb, D. P. Siddons, K. Sokolowski-Tinten, Th. Tschentscher, D. von der Linde, and J. B. Hastings, Science 315, 633 (2007).

${ }^{24}$ M. Cornacchia, J. Arthur, L. Bentson, R. Carr, P. Emma, J. Galayda, P. Krejcik, I. Lindau, J. Safranek, J. Schmerge, J. Stohr, R. Tatchyn, and A. Wootton, SLAC-PUB Report No. 8950, 2001.

${ }^{25}$ J. Isberg, J. Hammersberg, H. Bernhoff, D. J. Twitchen, and A. J. Whitehead, Diamond Relat. Mater. 13, 872 (2004).

${ }^{26}$ F. Scholze, H. Henneken, P. Kuschnerus, H. Rabus, M. Richter, and G. Ulm, Nucl. Instrum. Methods Phys. Res. A 439, 208 (2000).

${ }^{27}$ J. Isberg, M. Gabrysch, A. Tajani, and D. J. Twitchen, Semicond. Sci. Technol. 21, 1193 (2006). 